\title{
Rechtssprache in der Übersetzerausbildung
}

\author{
Blanka JENČÍKOVÁ
}

\begin{abstract}
Legal language in translator training

The topic of this paper is legal language and its integration into the teaching process in the training of translators working in the language combination German / Slovak; this area of didactics is relatively unexplored in Slovakia. The main aim of the study is to emphasize the importance of developing professional translation competence as part of translator training, as this competence is of significant assistance when creating content-correct translations. Based on an analysis of student translations, the article lists the most common problems encountered when translating legal texts, mainly related to the use of incorrect translation strategies.
\end{abstract}

Keywords: legal translation, didactics, translation competence, translator training

DOI: doi.org/10.15452/StudiaGermanistica.2020.27.0009

\section{Einleitung}

Die rasche Entwicklung in allen Fachbereichen stellt nicht nur den Übersetzer- und Dolmetscherberuf, sondern auch den Übersetzungs- und Dolmetschunterricht vor neue Herausforderungen. Die eindeutige Vorherrschaft der Fachübersetzung über die literarische Übersetzung auf dem Übersetzungsmarkt zeigt eindeutig die Richtung, in welche sich die Übersetzerausbildung bewegen soll und muss. Neben der Vermittlung einer allgemeinen translatorischen Kompetenz werden zurzeit in die universitären Translatorencurricula immer öfter bestimmte Fachgebiete aufgenommen, meistens als Teil- oder Ergänzungsfach. In dem Beitrag soll an einem konkreten Beispiel des auf die Rechts- und Wirtschaftssprache gerichteten Studienprogramms dargelegt werden, wie ein Sachfach im Rahmen der Ausbildung von Übersetzern behandelt werden kann. Einleitend wird die Rechtssprache definiert und im weiteren Teil wird auf das Thema des im Rahmen der Übersetzerausbildung zu erwerbenden Fachwissens näher eingegangen. Abschließend werden einige Überlegungen zur möglichen didaktischen Handhabung der Rechtssprache im Rahmen der Übersetzungsübungen in der Sprachkombination Deutsch - Slowakisch vorgestellt, wobei hier das Hauptaugenmerk auf die Vermittlung der juristischen Fachterminologie und der für die Sprachkombination Deutsch - Slowakisch relevanten Übersetzungsprobleme und Übersetzungsstrategien gerichtet wird. 


\title{
2. Rechtssprache
}

\subsection{Rechtssprache im Kontext der Gemein- und Fachsprache}

Den Gegenstand der Untersuchungen dieses Beitrags bildet die Rechtssprache in der Übersetzung und als Inhalt der universitären Curricula in der Übersetzerausbildung. Aus diesem Grund wird einleitend das Verhältnis zwischen der Gemein-, Fach- und Rechtssprache erläutert.

\begin{abstract}
„Als Gemeinsprache wird in der Regel die umfassende Sprache, die die allgemeinen Bereiche der Welt thematisiert bezeichnet.... Fachleute bedienen sich primär bei ihrer Kommunikation der Gemeinsprache, die Fachkommunikation verlangt allerdings wesentlich mehr Präzision, weshalb fachspezifischen Begriffen vom Fachkollektiv eine eindeutige Bedeutung zugeordnet wird, oft in Form von expliziten Definitionen, die von jeweiligen Fachleuten über Konsens formuliert werden." (Kadric 2012:153-154)
\end{abstract}

In ihrer Definition hebt Mira Kadric die gegenseitige Beeinflussung der Gemeinsprache und der Fachsprachen hervor und die Ausrichtung der Fachsprachen auf spezifische Zwecke, die sie erfüllen (vgl. Stolze 1999:22). Fachsprache ist ohne Gemeinsprache also nicht denkbar und es heißt auch, dass jeder fachsprachliche Text, jede fachsprachliche Äußerung eine mehr oder minder starke Bindung an die Gemeinsprache aufweist (Patocka 1999:6-7). Außerdem ist nach Theodor Ickler die Beeinflussung in umgekehrter Richtung zu beobachten, wovon die sog. Amateursprache zeugt. Da die meisten Menschen darüber hinaus in irgendwelchen Fächern tätig sind, übertragen sie einen Teil ihrer dort geltenden Sprachgewohnheiten in den nichtfachlichen Alltag (Ickler 1997:91).

Die Rechtssprache erfüllt laut Pavel Holländer (1995:329-330) die regulative Funktion als Sprache der Rechtsnormen und die informative Funktion als Sprache, mit welcher der Gesetzgeber den Adressaten der Rechtsnormen die Arten des obligatorischen bzw. erlaubten Verhaltens bekannt gibt. Aus den angeführten Funktionen der Rechtssprache ergeben sich dann die Merkmale der Rechtssprache. Die regulative Funktion erfordert die Eindeutigkeit, d. h. das Ausschließen der Synonymie, und die Genauigkeit. Als ein Mittel der Exaktheit gilt die Formalisierung sowohl auf der lexikalischen Ebene als auch auf der stilistischen Ebene. So ist die juristische Fachsprache durch Formalien geprägt, wie bestimmte Wendungen oder feststehende Redeformeln (Bsp.: Im Namen des Volkes), die oft einen archaischen Charakter aufweisen. Die informative Funktion der Rechtssprache hängt eng mit der Forderung ihrer Verständlichkeit zusammen, insofern zu Adressaten nicht nur Juristen, sondern auch Rechtsbefolger zählen. Unter Kaiserin Maria Theresia musste jedes neue Gesetz einem buta ember, einem einfachen Mann, vorgelesen werden. Erlassen durfte es nur werden, wenn er es verstand.

Das Spezifikum der Rechtssprache beruht in ihrer Bindung an ein konkretes Rechtssystem. So kann von einer einheitlichen Fachsprache des Rechts höchstens als Abstraktion gesprochen werden. Die Rechtsordnung bestimmt alle kommunikativen Parameter, von der oder den verwendeten Sprachen über die Begriffe und Benennungen bis hin zu einzelnen Textsortenkonventionen. In diesem Sinne kann etwa im deutschsprachigen Raum nur von einer Rechtssprache Deutschlands, einer Rechtssprache Osterreichs oder einer Rechtssprache der Schweiz gesprochen werden (de Groot 1991:283 zit. nach Sandrini 1999:12).

\subsection{Rechtssprache im Kontext des Übersetzungsunterrichts}

Im Gegensatz zu Deutschland, wo die Didaktik der Fachübersetzung relativ tiefgehend untersucht wird (z. B. Daum 2003, Kautz 2002, Nord 2010), spürt man in der Slowakei immer noch deutliche Lücken im Bereich der didaktisch-theoretischen Handhabung der Fachübersetzung und somit auch der Rechtsübersetzung (vgl. Wrede 2016:59). Da infolge der zunehmenden Globalisierung und besonders im europäischen Raum bedingt durch die Entstehung und Entfaltung der Europäischen Union der Bedarf an der sprachlich vermittelten Rechtskommunikation rasch und ständig wächst, 
ist es von großem Interesse Untersuchungen im Bereich der Rechtsübersetzung allgemein und ebenfalls in der Didaktik im Rahmen der Übersetzerausbildung vorzunehmen.

Bei der Übersetzung von Rechtstexten geht es nicht nur um den Transfer aus der Fachsprache einer Ausgangssprache in die Fachsprache einer Zielsprache, sondern in der Regel um die Übertragung von Inhalten einer Rechtsordnung zur Verwendung in einer ganz anders gearteten Rechtsordnung (Daum 2003:38). In diesem Zusammenhang stellt sich die Frage, wie die Rechtsübersetzer am besten auszubilden sind. Ist es besser Juristen die Fremdsprachenkenntnisse oder lieber Linguisten das Rechtswissen beizubringen? Meines Wissens gibt es im Rahmen der universitären Übersetzerausbildung zumindest in der Slowakei keine einheitlichen Lehrpläne. Jeder Lehrstuhl oder jedes Institut passt die Curricula eigenen Bedürfnissen an, wobei die Studienprogramme üblicherweise auf mehrere Fachbereiche gerichtet sind, damit die Studenten ein möglichst breit gefächertes Allgemein- und Fachwissen erhalten.

Ein speziell auf die Rechts- und Wirtschaftssprache orientiertes Studienprogramm für Übersetzer gibt es an der P. J. Šafárik-Universität in Košice, dessen Ziel in der Ausbildung professioneller Übersetzer und Dolmetscher für europäische und slowakische Institutionen besteht. Bereits in der Bachelorstufe absolvieren die Studenten Vorlesungen und Seminare in der slowakischen Sprache zu Recht und Wirtschaft und parallel dazu laufen Übersetzungsübungen in der deutschen, englischen und französischen Sprache - je nach der betreffenden Sprachkombination. Im Bereich des Rechts werden das bürgerliche Recht, das Strafrecht und das Handelsrecht eingehender in separaten Veranstaltungen je ein Semester lang behandelt, wobei als Wahlfächer mitunter andere Rechtsgebiete angeboten werden. Fast für alle Studenten, bei denen es sich überwiegend um Absolventen von Gymnasien, Handelsakademien oder sonstigen Fachmittelschulen mit Abiturabschluss handelt, ist Recht ein völlig neues Gebiet nicht nur in der Fremdsprache, sondern auch in ihrer Muttersprache. Somit erwiesen sich die slowakischen Rechtsvorlesungen und Seminare während des gesamten Bachelorstudiums als effiziente Unterstützung zu fremdsprachlichen Übersetzungsübungen. Die Kenntnis von allen an der Rechtskommunikation beteiligten Rechtssystemen ist nämlich die Voraussetzung für die kontrastive Behandlung der Rechtsterminologie und somit für die Arbeit mit Rechtstexten. Das erworbene juristische Fachwissen wirkt unterstützend sowohl beim Textverständnis allgemein, als auch bei der Ausgangstextanalyse, der Entwicklung der Recherchekompetenz und der Lösung von Übersetzungsproblemen. Hauptsächlich im Bereich der Rechtsterminologie heißt es nämlich ständig zu vergleichen. Der Übersetzer muss die genaue Bedeutung des betreffenden Terminus in der Ausgangssprache, d. h. in der Rechtsordnung des betreffenden Landes, feststellen und danach einen entsprechenden Terminus in der Zielsprache, d. h. in den Rechtsvorschriften des Landes der Zielsprache, suchen oder eine adäquate Übersetzungsstrategie wählen.

\section{3. Überlegungen zur Gestaltung des Übersetzungsunterrichts}

Als mir vor einiger Zeit als praktizierender Übersetzerin und Dolmetscherin angeboten wurde, Übersetzungsübungen zu unterrichten, war es für mich eine große Herausforderung. Nach den ersten Versuchen mit der Übersetzung eines Gerichtsurteils, mit welcher ich trotz einer umfangreichen Analyse des Ausgangstextes im Unterricht meine Studenten und anschließend bei der Korrektur auch mich selbst gequält hatte, beschloss ich, eine Erfolgsstrategie zu entwickeln, nämlich einen effizienten und systematischen Plan für praktische Übersetzungsübungen, der zur erfolgreichen Ergebnissen führen würde. Mein Ziel wird vielleicht am besten mit dem folgenden Zitat von Ulrich Kautz (2002:47) ausgedrückt:

„Der Übersetzungsunterricht ist nicht dazu da, gute Übersetzungen hervorzubringen, sondern gute Übersetzer. Das bedeutet, wir müssen prozess- und nicht in erster Linie ergebnisorientiert unterrichten - wir müssen den Lernern eine Strategie der Lösungen von wesentlichen (nicht: allen!) Problemen, die beim Übersetzen auftreten, bewusst machen. “ 
Zur Erstellung eines zweckmäßigen und sinnvollen Lerninhalts einer Lehrveranstaltung ist es notwendig, die Lernziele und die zur Verfügung stehenden Lernmittel zu identifizieren. Demnach habe ich zuerst die zu erreichenden Lernziele festgelegt, damit ich anschließend unter Berücksichtigung der Ausgangssituation eine passende Strategie zum Erreichen dieser Ziele entwickeln konnte.

Das Hauptziel des Übersetzungsunterrichts beruht in der Entwicklung der translatorischen Kompetenzen, also hauptsächlich der Sprachkompetenz, der interkulturellen Kompetenz, der Fachkompetenz, der Technikkompetenz und der Übersetzungskompetenz (vgl. Krajčovičová 217:77 ff.), um den Studenten im Sinne des vorstehend angeführten Zitats ein Instrument zur Lösung von wesentlichen Übersetzungsproblemen in die Hand zu geben. Allerdings bereits bei der ersten Kompetenz - der Sprachkompetenz - stieß ich auf ein Problem. Für den Umgang mit Rechtstexten und eine progressive Entwicklung der erwähnten Kompetenzen wäre das Niveau C1 des europäischen Referenzrahmens erforderlich. Durch eine politische Entscheidung wurde aber Deutsch im slowakischen Schulwesen zur zweiten Fremdsprache mit einer wesentlich niedrigeren Stundendotation als Englisch gemacht. Somit wird beim Abitur nur Niveau B1 gefordert. Seit 2019 können die slowakischen Schüler wieder eine andere Sprache als Englisch als erste Fremdsprache wählen, doch es wird ein paar Jahre dauern, bis sich die Situation wieder einrenkt. So ist diese Tatsache bei der Gestaltung des Lerninhalts zu berücksichtigen und es muss auf den vorhandenen Kenntnisstand angeknüpft werden, sonst würde man die Studenten überfordern, was den Verlust der Motivation und des Interesses am Studium zur Folge hätte.

In dem Sinne war der einzige Weg vor der Übersetzung eine gründliche terminologische Vorbereitung für die einzelnen Themenbereiche mit Hilfe von didaktisch angepassten Texten, terminologischen Übungen und gesteuerten Diskussionen unterstützt durch aktive Hausarbeit durchzuführen. Neben der Erweiterung der Sprachkenntnisse bezweckt diese Phase der Behandlung des jeweiligen Themas sowohl die Entwicklung des Textverständnisses, der Recherchekompetenz, der Arbeit mit Paralleltexten, der Komparationsfähigkeit, als auch die aktive Anwendung der Rechtsterminologie nicht nur in der Mutter- sondern vor allem in der Fremdsprache. Als zweiter Schritt folgt dann die Übersetzung von didaktisch angepassten realen Texten zuerst in die Muttersprache und anschließend im dritten Schritt in die Fremdsprache, jeweils mit gründlicher Ausgangstextanalyse und Vorbereitung vor der Transferphase und anschließender Evaluierungsphase in Form von Diskussion über die Übersetzungsprobleme, die angewendeten Verfahren und die aufgetretenen Fehler. So wurden im Bereich des Strafrechts beispielsweise die Phasen des Erkenntnisverfahrens, die Arten der Straftaten oder die deutsche Strafprozessordnung mit dem Verlauf einer Gerichtsverhandlung behandelt. Erst durch diese gründliche Entwicklung der Sprach-, Fach- und Recherchekompetenz waren die Studenten fähig, sinnvolle Translate von didaktisch angepassten Texten aus der Übersetzungspraxis zu erstellen, und das sogar in die Fremdsprache. Die aktive Beherrschung nicht nur der slowakischen, sondern auch der deutschen Fachterminologie ist ebenfalls für die Dolmetschübungen von großer Bedeutung, die später im Rahmen des Studiums auf die Übersetzungsübungen folgen, und nicht zuletzt für die übersetzerische Praxis, wo auf dem slowakischen Übersetzungsmarkt in der Sprachkombination Deutsch - Slowakisch marktbedingt in beide Richtungen übersetzt wird. Bei den beglaubigten Übersetzungen von Gerichtsurkunden geht es sogar überwiegend um die aktive Übersetzung in die Fremdsprache. Aus dem Grund ist dieser Aspekt bei der Übersetzerausbildung zwingend zu berücksichtigen.

\section{3. Übersetzungsprobleme bei der Übersetzung von Rechtstexten aus dem Deutschen ins Slowakische}

\subsection{Wahl der zu übersetzenden Texte}

Im weiteren Teil meines Beitrags würde ich mich gern didaktisch relevanten Aspekten bei der Behandlung von Rechtstexten im Übersetzungsunterricht und der Lösung von Übersetzungsproblemen widmen. An konkreten Beispielen einer Gerichtsurkunde zeige ich, mit welchen Schwierigkeiten 
die Studenten bei der Rechtsübersetzung aus dem Deutschen ins Slowakische zu kämpfen haben und welche Bereiche aus der didaktischen Sicht in dieser Sprachkombination mehr Aufmerksamkeit verdienen.

Zur Analyse wählte ich einen Strafbefehl in Form einer realen durch das Amtsgericht Cham erlassenen Urkunde mit Stempelabdrücken und Unterschriften, die allerdings für die didaktischen Zwecke anonymisiert wurde. Da ihre Länge etwa eine Normseite beträgt, war keine Kürzung erforderlich. Eine Normseite, also 1800 Zeichen inklusive Leerzeichen, erwies sich in meiner Praxis als sinnvoller Umfang für Übersetzungsaufträge im Unterricht. Zu lange Texte erschweren und verlängern nämlich unnötig die Evaluierungsphase. Die analysierten Übersetzungen wurden von Studenten des zweiten und des vierten Semesters der Bachelorstufe des bereits vorstehend erwähnten Studienprogramms im Rahmen der Übersetzungsübung zu Strafrecht im Laufe des Semesters übersetzt. Insgesamt wurden der Analyse 17 Übersetzungen unterzogen.

\section{2. Übersetzungsvorbereitende Analyse im Unterricht}

Vor der eigentlichen Übersetzung, also der Transferphase, die in der Regel als Hausarbeit vergeben wird, erfolgt im Unterricht eine eingehende Analyse des Ausgangstextes, ergänzt um die Arbeit mit Paralleltexten. Im Sinne der funktionalen Übersetzung und der Skoposerfüllung, wird jede Übersetzung im Unterricht als realer Übersetzungsauftrag erteilt, um den Zweck, die Adressaten und die Kultureinbettung ermitteln zu können. An dieser Stelle muss aber zugleich bemerkt werden, dass die slowakische Übersetzungskultur in dieser Hinsicht wesentliche Lücken aufweist. Die meisten Übersetzungsaufträge werden nicht näher spezifiziert, man bekommt üblicherweise nur eine E-Mail mit der Bitte um Übersetzung der angehängten Datei (vgl. dazu auch Tomášiková 2013:68). Die Studenten sollten den Strafbefehl für den Angeklagten übersetzen, der diese Gerichtsentscheidung per Post erhalten hat und seinen Inhalt nicht versteht. Anhand dieser Information und des erworbenen Fachwissens konnte im Unterricht eine eingehende Ausgangstextanalyse gemacht werden.

In Bezug auf die Bindung der Rechtssprache auf ein konkretes Rechtssystem, ist die Information über Deutschland als Herkunftsland dieses Dokuments von großer Bedeutung, sowie die Zuweisung zum konkreten Rechtsbereich in dem gegebenen Rechtssystem, in diesem Fall dem Strafprozessrecht. Die Abgrenzung des Strafrechts gegenüber vom bürgerlichen Recht ermöglicht zum Beispiel die richtige Identifizierung der am Verfahren beteiligten Parteien oder der Bezeichnung von Gerichtsurkunden, was zur Findung von richtigen Äquivalenten der Rechtstermini beiträgt. Ein typischer Fehler bei mangelnden Fachkenntnissen ist die Verwechslung von Klage und Anklage, d. h. ,žaloba' und ,obžaloba', wo der erste Begriff dem bürgerlichen Recht angehört und der zweite wieder im Strafrecht verwendet wird.

Ein schriftlicher Strafbefehl wird in Deutschland im Rahmen eines Strafbefehlsverfahrens erlassen, bei welchem es sich um ein vereinfachtes Verfahren zur Bewältigung leichter Kriminalität handelt. Der Antrag wird von der Staatsanwaltschaft vor der Eröffnung der mündlichen Hauptverhandlung gestellt und die Entscheidung obliegt dem Strafrichter. Bei einem Strafbefehlsverfahren können nur sogenannte Vergehen, prečiny‘ geahndet werden. Im Gegensatz zu Verbrechen ist der Strafrahmen bei einem Vergehen unterhalb eines Jahres. Mögliche Rechtsfolgen sind außerdem zum Beispiel: Geldstrafe, Verwarnung, Fahrverbot, Verfall, Entziehung der Fahrerlaubnis oder Absehen von Strafe. Das Strafbefehlsverfahren ist im 6. Buch der deutschen Strafprozessordnung (§§ 407-412) geregelt (URL 1). Nachdem die Staatsanwaltschaft den Erlass eines Strafbefehls beantragt hat, wird der Beschuldigte als Angeschuldigter bezeichnet und durch den Erlass eines Strafbefehls wird er schließlich zum Angeklagten. Als Rechtsmittel kann ein Einspruch eingelegt werden. In der Slowakei wird ein Strafbefehl ,trestný rozkaz' vom Einzelrichter ,samosudca' in der Hauptverhandlung vor der Eröffnung der öffentlichen Verhandlung erlassen. Den Strafrichter als Spruchkörper kennt das slowakische Justizsystem nicht. Die Rechtsfolgen sind ähnlich wie in Deutschland, allerdings kann in der Slowakei durch den Strafbefehl eine Freiheitsstrafe bis zu 3 Jahren auferlegt werden. Der Täter wird in einem Strafbefehl als obvinený,Angeschuldigter ' bezeichnet 
und das Rechtsmittel heißt odpor ,Einspruch“. Geregelt wird das gesamte Verfahren in $\S \S 353 \mathrm{ff}$. des slowakischen Gesetzes Nr. 301/2005 Slg. Strafprozessordnung, Trestný poriadok‘ (URL 2).

Bei der Arbeit mit Paralleltexten wurde die Aufmerksamkeit zuerst der Makrostruktur des Textes gewidmet und anschließend wurde die terminologische, syntaktische und stilistische Ebene beider Sprachvarianten kontrastiv behandelt. Die Studenten wurden auf die Wahl des richtigen Übersetzungstyps im Sinne der Definition von Christiane Nord (2010:51-52) hingewiesen. Sie unterscheidet zwei Grundtypen - die dokumentarische und die instrumentelle Übersetzung. Bei der dokumentarischen Übersetzung wird eine Kommunikationshandlung in der Ausgangskultur dokumentiert und der zielkulturelle Empfänger steht wie ein Beobachter außerhalb des Geschehens. Die Übersetzung ist hier im Gegensatz zur instrumentellen Übersetzung als solche erkennbar. Die instrumentelle Übersetzung funktioniert wieder als eigenständiges Kommunikationsinstrument in einer zielkulturellen Situation. Im Falle der studentischen Übersetzungen geht es darum, den Inhalt der von deutschen Gerichten erlassenen Urkunden dem slowakischen Rechtsanwalt bzw. dem slowakischen Angeschuldigten zu vermitteln, d. h. zu dokumentieren. Aus dieser Sicht erweist sich die dokumentarische Übersetzung als primärer Übersetzungstyp sinnvoll. Für den Übersetzer bedeutet es, dass in den Zieltext die Merkmale des Ausgangstextes übertragen werden, um die dokumentarische Vergleichbarkeit zu gewährleisten. Da es dem Zieltextempfänger klar ist, dass es sich um eine Übersetzung handelt, sind im Zieltext Anmerkungen und Erklärungen zulässig. Während aber in der Makrostruktur die dokumentarische Übersetzung anzuwenden ist, müssen die Studenten auch dafür sensibilisiert werden, dass in der Mikrostruktur die terminologischen Besonderheiten und die stilistischen Differenzen des Rechtsdiskurses in beiden Sprachen in Erwägung zu ziehen sind. Auf der syntaktischen und lexikalischen Ebene wird nicht empfohlen, streng dem Original treu zu bleiben (vgl. dazu auch Duricová 2017:64-67, Wrede 2016:73). Es wird dadurch die Verständlichkeit im Sinne der bereits vorstehend erwähnten informativen Funktion der Rechtssprache unterstützt.

\subsection{Analyse von studentischen Translaten}

Nach einer tiefgehenden Analyse des Ausgangstextes und der komparativen Arbeit mit Paralleltexten sollten die Studenten zu Hause Translate anfertigen. Der Strafbefehl wurde zur Übersetzung als eingescannte PDF-Datei vergeben. Da es sich um eine Kopie des Originaldokuments mit Stempelabdrücken und eigenhändigen Unterschriften mit einer relativ komplizierten Textstruktur handelt, kam es zu vielen Auslassungen im Zieltext. Die Stempelabdrücke im Kopf des Strafbefehls enthalten Informationen über die Zustellung und Rechtskräftigkeit der Urkunde. Am Ende des Dokuments befinden sich wieder das Ausstellungsdatum, der Stempel des ausstellenden Gerichts und die Unterschriften von zuständigen Richtern oder sonstigen Justizangestellten. Indem diese Teile wichtige Informationen enthalten, werden sie in der slowakischen Übersetzungspraxis, besonders bei beglaubigten Übersetzungen, streng dokumentarisch übersetzt. Alle Stempelabdrücke werden als solche gekennzeichnet, und der Text wird ebenfalls mitübersetzt. Wobei sie oft auch näher beschrieben werden, falls sich zum Beispiel in der Mitte ein Staatswappen befindet. Ähnliches gilt auch für die Unterschriften und handschriftlich eingefügte Textteile. Einige Studenten haben die Stempelabdrücke als Abbildungen in der PDF-Datei ausgeschnitten und diese dann unübersetzt in den Zieltext übertragen und einige haben nur angemerkt, dass sich an der betreffenden Stelle ein Stempel befindet, ohne den Text des Stempels zu erwähnen. Bei einigen fehlten wieder das Ausstellungsdatum und die Unterschriften am Ende des Dokuments. Ein weiteres Problem stellten die Hervorhebungen im Text durch Fettschrift oder Unterstreichung dar. Sie wurden in vielen Fällen ignoriert oder nicht durchgehend verwendet.

Bei der anschließenden Diskussion im Unterricht hat sich herausgestellt, dass mehrere Studenten technische Probleme mit der Erstellung des 1:1 Formats im Word hatten, was auf mangelnde Fertigkeiten im Umgang mit Texteditoren deutet. Obwohl heute auf dem Übersetzungsmarkt die Tendenz zum allmählichen Übergang auf CAT-Tools zu beobachten ist (hauptsächlich bei technischen Texten), werden die Gerichtsurkunden in der Slowakei immer noch in Papierform oder als 
eingescannte PDF-Dateien zur Übersetzung geschickt, so ist auch diese technische Kompetenz von Wichtigkeit. Durch eine möglichst getreue Übertragung der formalen Struktur des Ausgangstextes in den Zieltext wird nämlich bessere Vergleichbarkeit und Orientierung im Text sichergestellt.

Bei der inhaltlichen Struktur des Spruchteils des Strafbefehls kam ebenfalls die Anwendung einer falschen Übersetzungsart zum Vorschein. Der Ausgangstext hat folgende Grundstruktur:

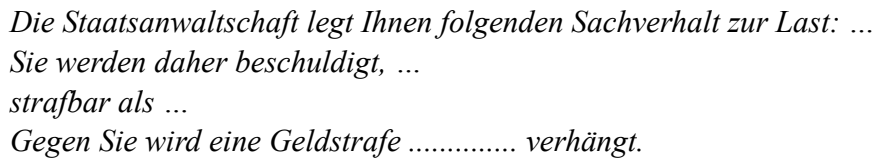

Die Struktur der als Paralleltexte verwendeten slowakischen Strafbefehle ist wie folgt:

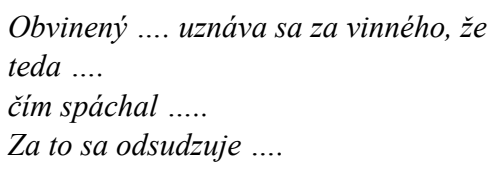

Wohl unter dem Einfluss von Paralleltexten haben mehrere Studenten versucht, die Informationen aus dem deutschen Ausgangstext in die slowakische Textstruktur zu übertragen. Zwar sind die Strafbefehle in beiden Sprachen inhaltsgemäß fast identisch, während aber der deutsche Strafbefehl als ein Schreiben an den Angeschuldigten formuliert wird und der Angeschuldigte in der 3. Person Plural als Empfänger des Schreibens angesprochen wird (Sie werden ... beschuldigt), wird der slowakische Strafbefehl als eine gerichtliche Entscheidung formuliert, in welcher die 3. Person Singular verwendet wird (Obvinený .... uznáva sa vinného). Durch den Versuch um den Wechsel der grammatischen Kategorie (3. Person Plural in 3. Person Singular) infolge der zielsprachig orientierten Übersetzung wurden unnötige Fehler und Bedeutungsverschiebungen generiert. Als Beispiel führe ich den ersten Satz des Spruchteils an:

Die Staatsanwaltschaft legt Ihnen folgenden Sachverhalt zur Last:

(1) Prokurátor vám ukladá nasledujúce skutočnosti, že obvinený:

(2) Obvinený: Rudolf Suchý, narodený 30.03.1962 v Považskej Bystrici je vinný, že

(3) Obvinený je vinný, že ....

In allen drei angeführten Beispielen bemühten sich die Studenten die 3. Person Singular anzuwenden und somit wurden sie gezwungen analog zum slowakischen Strafbefehl den Begriff obvinený,Angeklagter' zu verwenden, der übrigens in dem gesamten Ausgangstext überhaupt nicht vorkommt, um die Kohärenz auf der Textebene zu bewahren. Allerdings ist dadurch eine wichtige Information verloren gegangen, nämlich dass der Sachverhalt von der Staatsanwaltschaft zur Last gelegt wird. In dem ersten Beispiel befindet sich zwar Prokurator ,Staatsanwalt', wohl in der Bemühung um Beibehaltung aller Informationen, doch ist auf diese Weise ein schwerwiegender Bedeutungsfehler entstanden. Im zweiten Beispiel gibt es außerdem hinzugefügte Informationen, die der Übersetzer von einem anderen Textteil höchstwahrscheinlich unter Einfluss des slowakischen Paralleltextes übertragen hat. Die Anwendung der instrumentellen Übersetzung durch die Übernahme der zielkulturellen Textstruktur in den Zieltext führte also zu erheblichen Fehlern und Bedeutungsverschiebungen infolge der Auslassungen oder Hinzufügungen im Text. Die Tatsache, dass es für Studenten schwierig ist zu entscheiden, wann dokumentarisch und wann instrumental vorzugehen ist, beweist ferner das bei der Anrede und dem Namen in der Adresse des Adressaten angewendete Übersetzungsverfahren:

$\begin{array}{ll}\text { Herrn } & \text { Pánovi } \\ \text { Roland Suchy } & \text { Rolandovi Suchému } \\ \text {... } & \ldots .\end{array}$

Die angeführte Übersetzung ist wortwörtlich, wobei aber in diesem Fall die Konventionen der Zielsprache in Betracht gezogen werden sollten. Also die Anrede und der Name im Nominativ sollte lauten: Pán Roland Suchý. 
Auf der syntaktischen Ebene wurde auf mehreren Stellen das Verb werden falsch identifiziert, wodurch es zur Verwechslung von Passiv Präsens und Passiv Futur kam:

Gegen Sie wird eine Geldstrafe .......... verhängt.

(1) Bude Vám uložený peňažný trest .... (Passiv Futur)

(2) Je Vám uložený peňažný trest .... (Zustandspassiv Präsens)

Dieser Strafbefehl wird rechtskräftig und vollstreckbar ...

(1)Tento trestný rozkaz bude právoplatný a vykonatelný (Futur)

Im Spruchteil (Rubrum) und in sonstigen Teilen von slowakischen Gerichtsentscheidungen, welche die jeweilige Entscheidung des Gerichts zum Ausdruck bringen, werden die reflexiven Formen von Verben im Präsens bevorzugt, in dem oben angeführten Fall wird demnach die zielsprachig orientierte Übersetzung empfohlen: Ukladá sa Vám peňažný trest ... bzw. Tento trestný rozkaz sa stáva právoplatným a vykonatelným.

Im Bereich der Lexik wurde die strafrechtliche Terminologie in den meisten Zieltexten richtig angewendet. Bei den Begriffen wie Strafbefehl, fahrlässig, rechtskräftig, Geldstrafe oder Einspruch wurden bis auf einige Ausnahmen richtige zielsprachige Äquivalente gewählt, was auf die bereits erwähnte terminologische Vorbereitung zurückzuführen ist. Probleme traten allerdings bei der Terminologie aus dem verkehrspolizeilichen Bereich auf. Beim Kompositum Kennzeichen ist einerseits die exotisierende wortwörtliche Übersetzung zu finden: poznávacia značka, die zwar umgangssprachlich gebräuchlich ist, in verkehrsrechtlichen Vorschriften aber in der Bedeutung des Länderkennzeichens SK angewendet wird, andererseits die adaptierende Übersetzung allerdings unter Verwendung des veralteten Akronyms $\breve{S} P Z$, das in den slowakischen Rechtsvorschriften nicht mehr verwendet wird. Die empfohlene übersetzerische Lösung in diesem Fall wäre die adaptierende Übersetzung, die in der zielsprachlichen Kultur gebräuchlich ist, entweder als Akronym $E \check{C} V$ oder ungekürzt als evidenčné čislo (vozidla). Ferner wurde in vielen Fällen die Fahrerlaubnis mit dem Führerschein verwechselt und in dem Zusammenhang wurde ebenfalls die Klasse der Fahrerlaubnis als trieda übersetzt, wobei im Slowakischen der Begriff skupina verwendet wird. Aus dem Angeführten ergibt sich, dass sowohl auf der syntaktischen als auch auf der lexikalischen Ebene die Verständlichkeit des Zieltextes für seinen Empfänger im Vordergrund stehen sollte, was durch die Berücksichtigung der in der Zielsprache üblichen Konventionen zu erreichen ist.

Eine weitere Gruppe von lexikalischen Fehlern bilden umgangssprachliche Ausdrücke wie šoférovat', dokopy, behom, udelit' trest, die zur stilistischen Absenkung führen. Bei der Übersetzung ist der Stil des Ausgangstextes, der in diesem Fall durch die juristische Bildung seines Produzenten und die Textsorte determiniert wird, zu bewahren. Außerdem wurden während der Analyse Tschechismen (odstavec, prehlásenie, za účelom) und unter Einfluss der Ausgangssprache entstandene Interferenzfehler (nemali ste $k$ tomu, $k$ riadeniu ... by potreboval) vorgefunden, die zwar keinen Einfluss auf die Bedeutung nehmen, die sprachliche Qualität des Translats aber beeinträchtigen. Daher sind die Studenten zur Vermeidung von Fehlern dieser Art anzuhalten.

Standardformeln, die für die Rechtssprache typisch sind, stellten ebenfalls eine Fehlerquelle dar. Fahren ohne Fahrerlaubnis wurde von allen Studenten ausgangssprachlich orientiert übersetzt, und zwar entweder wortwörtlich jazdenie bez vodičského oprávnenia oder sogar durch Konkretisierung und Hinzufügung von zusätzlichen, im Ausgangstext nicht vorkommenden Informationen prečin riadenia súpravy vozidiel bez adekvátneho vodičského oprávnenia übersetzt. Standardisierte Formeln dienen zur Vereinfachung interner Informationen im juristischen Verkehr. Der Übersetzer hat bei solchen syntaktischen Konstruktionen keine Freiheit und muss die in der Zielsprache übliche Konstruktion verwenden (vgl. Ďuricová 2017:66). In dem angeführten Fall wäre es vedenie (motorového) vozidla bez príslušného vodičského oprávnenia.

Bei der Übersetzung von geographischen Namen gilt der Grundsatz, dass diese vom Original zu übernehmen sind, falls in der Zielsprache kein gebräuchliches Äquivalent zur Verfügung steht (vgl. Duricová 2016:109). Außerdem ist bei der Wahl der richtigen Übersetzungsstrategie zu beachten, an welcher Stelle im Text sich der betreffende Name befindet, da die Adressen zum Bei- 
spiel unverändert vom Ausgangstext zu übernehmen sind. Die Studenten behielten Nürnberg in der Adresse des Zustellungsberechtigten richtig in der Originalform, obwohl es im Slowakischen das standardisiertes Äquivalent Norimberg gibt. Bei Furth im Wald hatte aber etwa die Hälfte von ihnen diesen Ausdruck nicht als einen geographischen Namen erkannt oder auch übersetzt: Brod nad lesmi, v lese, na lesnej ceste.

Die Behandlung von Akronymen in der Übersetzung ist ebenfalls ein oft diskutiertes Thema. Es herrschen verschiedene Ansichten, wie in diesem Fall vorzugehen ist. Man kann ein Akronym einfach in Originalform in die Zielsprache übernehmen, und zwar mit oder ohne Explikation in Klammern, oder eine andere Möglichkeit wäre im Zieltext die vollständige Übersetzung des Akronyms zu verwenden. Die Wahl eines geeigneten Übersetzungsverfahrens hängt von der gegebenen Kommunikationssituation und dem erwarteten Ziel ab. Bei der Übersetzung von internen Dokumenten einer Gesellschaft werden zum Beispiel die Bezeichnungen von einzelnen Managerpositionen im Original behalten $(A L G, G L, C E O)$, da diese hauptsächlich bei internationalen Konzernen in allen Ländern einheitlich und vor allem gesellschaftsspezifisch verwendet werden und allen Textempfängern bekannt sind. Durch ihre Übersetzung oder sogar durch Bildung von entsprechenden slowakischen Versionen von Akronymen käme es zu Kommunikationsstörungen. Ein anderer Fall sind aber die Gesetzesbezeichnungen und die Amtsbezeichnungen der Polizei in Rechtstexten. Hier wird die Übersetzung des jeweiligen Akronyms entweder wortwörtlich oder bei Bedarf auch explikativ empfohlen, um das Textverständnis für den Adressaten zu unterstützen (vgl. Tomášiková 2014:47). Im Rahmen der Vorbereitung wurden mit Studenten die Akronyme und die möglichen Übersetzungsstrategien besprochen, die konkreten Bedeutungen sollten sie aber selbständig durch Recherchieren herausfinden und sich anschließend für eine Übersetzungsstrategie entscheiden. Die abgekürzte Gesetzesbezeichnung $S t V G$ wurde in den meisten Fällen richtig gelöst, ein wesentliches Problem stellten allerdings folgende Akronyme dar:

PHM Müller, GPS Furth im Wald

(1) PHM Müller, GPS Furth im Wald

(2) policajný štráżmajster Müller, GPS Furth im Wald

(3) šéf policajného zboru Müller, GPS Furth im Wald

PHM wurde entweder im Original behalten (1) oder höchstwahrscheinlich mit Hilfe von zugänglichen Online-Wörterbüchern als strážmajster übersetzt (2). In einem Fall wurde der Polizeihauptmeister sogar zum Polizeichef gemacht (3). GPS hat niemand übersetzt, obwohl man durch Recherchieren sehr einfach finden könnte, dass es sich um eine Grenzpolizeistation handelt. Da die deutschen Amtsbezeichnungen der Polizei mit jenen in der Slowakei nicht korrespondieren, ist es empfehlenswert, eine wortwörtliche Übersetzung vorzunehmen. In diesem Fall wäre also PHM als hlavný policajný majster zu übersetzen (vgl. Tomášiková 2014:44).

\subsection{Analyse nach der Transferphase}

Aus der didaktischen Sicht ist die Analyse von angewendeten Übersetzungsverfahren und entstandenen Fehlern von wesentlicher Bedeutung für die Steigerung der Qualität von studentischen Translaten und somit für die Progression im Unterricht. Aus eigenen Fehlern lernen ist eine alte und bewährte Methode, um in Zukunft wiederkehrenden Fehlern vorzubeugen. Doch noch besser ist, dazu auch aus Fehlern von anderen zu lernen. In diesem Sinne wurde nach der Übersetzung des Strafbefehls eine aktive Diskussion über die angewendeten Übersetzungsstrategien geführt. Die Studenten wurden sowohl mit falschen als auch mit guten Lösungen konfrontiert und sie sollten entscheiden, was zulässig ist oder was geändert werden sollte und ihre Entscheidung entsprechend begründen. Mit der Wahl eines realen reich strukturierten Textes aus der Übersetzungspraxis wollte ich den Studenten den Sinn der dokumentarischen Übersetzungsart näherbringen und die dünne Linie, welche diese Art von der instrumentellen Übersetzung trennt, zeigen. Außerdem bot dieser Text auch viele Möglichkeiten zur praktischen Erklärung der bei der Übersetzung von geographischen 
Namen, Gerichtsbezeichnungen, Amtsbezeichnungen der Polizei und sonstigen Akronymen sowie Standardformeln zu verwendenden Übersetzungsverfahren. Dank gründlicher Analyse nach der Übersetzung wurde ein Teil der Fehler abgebaut und später bei der Übersetzung einer Anklage und eines Urteils war eine Progression zu sehen.

\section{Zusammenfassung}

Die Einbindung verschiedener Fachbereiche in die Übersetzerausbildung ist zur Notwendigkeit geworden. Die Rechtssprache erfordert in dieser Hinsicht eine besondere Aufmerksamkeit, indem sie Spezifika nicht nur auf der terminologischen Ebene, sondern auch im Bereich der Syntax und der Stilistik aufweist. In diesem Beitrag wurden an einem konkreten Beispiel in der Sprachkombination Deutsch - Slowakisch die Schwierigkeiten dargelegt, denen die Studenten im Übersetzungsunterricht bei der Behandlung von Rechtstexten begegnen. Aus der Analyse der Translate ergab sich das Problem mit der Anwendung der für die gegebene Textsorte adäquaten Übersetzungsstrategien. Zum Schluss wurde noch die Wichtigkeit einer gründlichen Fehleranalyse nach der Transferphase im Unterricht betont.

\section{Literaturverzeichnis}

DAum, Ulrich (2003): Übersetzen von Rechtstexten. In: ScHubERT, Klaus (Hrsg.): Übersetzen und Dolmetschen, Modelle, Methoden, Technologie. Tübingen, S. 33-46.

De Groot, Gérard-René (1991): Recht, Rechtssprache und Rechtssystem - Betrachtungen über die Problematik der Übersetzung juristischer Texte. In: Terminologie \& Traduction. 3/1991, Luxembourg, S. 279-315.

Ďuricová, Alena (2017): Vom Recht zur Rechtssübersetzung. In: RAKšányová, Jana et al. (Hrsg.): Quo vadis, Rechtsübersetzung? Bratislava, S. 49-76.

HolläNDER, Pavel (1995): Paradox právneho jazyka. In: Kultúra slova, 1995/6, S. 328-333. Zugänglich unter: https://www.juls.savba.sk/ediela/ks/1995/6/ks1995-6.html [10.07.2020].

ICKLER, Theodor (1997): Die Disziplinierung der Sprache. Fachsprachen in unserer Zeit. Tübingen. KADRIC, Mira (2012): Basiswissen Translation. Wien.

KaUtz, Ulrich (2002): Handbuch Didaktik des Übersetzens und Dolmetschens. München.

KRAJČOvIČOvÁ, Lenka (2017): Kompetenzprofil für RevisorInnen. Masterarbeit. Hamburg.

NoRD, Christiane (2010): Fertigkeit Übersetzen. Ein Kurs zum Übersetzenlehren und -lernen. Berlin.

Patocka, Franz (1999): Fachsprachen. In: ERnst, Peter (Hrsg.): Einführung in die synchrone Sprachwissenschat. Wien, S. 1-16.

SANDRINI, Peter (1999): Translation zwischen Kultur und Kommunikation: Der Sonderfall Recht. In: SANDRINI, Peter (Hrsg.): Übersetzen von Rechtstexten. Fachkommunikation im Spannungsfeld zwischen Rechtsordnung und Sprache. Tübingen, S. 9-43.

Stolze, Radegundis (1999): Die Fachübersetzung. Türbingen.

ToMášiková, Slavomíra (2013). Übersetzung der Gerichtstexte. In: Zrkadlá translatológie I: Preklad ako nástroj komunikácie. Prešov, S. 64-77. Zugänglich unter: http://iptatpresov.weebly.com/ uploads/3/6/5/6/3656532/zbornik_a5_1_cast_final.pdf [10.07.2020].

Tomášiková, Slavomíra (2014). Preklàd právnych̆ textov z nemeckého do slovenského jazyka. Prešov.

Wrede, Olga (2016): Právny text z pohl'adu didaktiky odborného prekladu. In: XLinguae, Volume 9 Issue 1. Zugänglich unter: http://xlinguae.eu/2016_09_01_05.html [10.07.2020].

\section{Internetquellen:}

URL 1: Strafprozessordnung. https://www.gesetze-im-internet.de/stpo/_407.html_[10.07.2020].

URL2:Trestnýporiadok. https://www.slov-lex.sk/pravne-predpisy/-/SK/ZZ/2005/301/20160701\#paragraf-353 [10.07.2020]. 